\title{
Asymptotic Locality and the Structure of Local Internal Symmetries
}

\author{
LAWRENCE J. LANDAU
}

II. Institut für Theoretische Physik der Universität Hamburg

Received February 26, 1970

\begin{abstract}
Symmetries are investigated from the locar viewpoint. Using the Haag-Ruelle construction, the action of a local internal symmetry on the asymptotic states is determined. A condition of "asymptotic locality" is derived and used to show that the symmetry acts linearly and locally on the asymptotic fields. Within a field theoretical framework it is shown that the internal symmetry must commute with the Poincare group. The general structure of an internal symmetry is determined. The uniqueness of the representation of the Poincaré group is discussed, and a simple example of an infinite component field is given to indicate what occurs when there are infinitely degenerate particle multiplets.
\end{abstract}

\section{Introduction}

There has been renewed interest in recent years concerning the structure of symmetries, primarily due to the consideration of groups such as $S U(6)$, which contain particles of different spin within a single multiplet, or groups which might contain mass-splitting, that is, particles of different masses within a single multiplet. The question arises as to whether it is possible to combine the Poincaré group and another symmetry group in a non-trivial way [1].

In this article symmetry groups are considered from the local viewpoint $[2,3]$. To each region $R$ of space-time is associated a set of operators $B(R)$ representing the measurements or operations which can be performed with laboratory apparatus confined to the region $R$. If $R_{1}$ and $R_{2}$ are space-like separated regions, the condition of locality states that $B\left(R_{1}\right)$ commutes with $B\left(R_{2}\right)$ :

$$
\left[B\left(R_{1}\right), B\left(R_{2}\right)\right]=0 \quad \text { for } \quad R_{2} \subset R_{1}^{\prime}
$$

where $R_{1}^{\prime}$ denotes the region space-like to $R_{1}$. In the field theoretical framework locality is expressed by

$$
\left[\phi_{\alpha}(x), \phi_{\mu}(y)\right]=0 \text { for }(x-y)^{2}<0 .
$$

In such a local theory we expect a symmetry operation to reflect the local properties (1) or (2). Consider a true symmetry of the physical system, i.e. a symmetry operation represented by a unitary operator $G$ 
acting on the Hilbert space of physical states. This symmetry can be looked at in two ways, as an action on the states of the system or as an action on the observables. From the second viewpoint we expect the symmetry to transform the set of observables $B(R)$ associated with the region $R$ onto the set of observables associated with a region $R_{G}$ :

$$
G B(R) G^{-1}=B\left(R_{G}\right) .
$$

Now it has been shown by Zeeman [4] that the most general mapping of regions $R \rightarrow R_{G}$ which preserves the causal relations among regions is an extended Poincaré transformation and a dilatation. (The dilatations can be ruled out as physical symmetries because they lead to unphysical mass spectra.) Thus we expect that the most general action of a local symmetry can be described as an extended Poincaré transformation followed by a transformation which maps the set of observables of each region onto itself. We restrict our attention to these latter symmetries which leave each region invariant. These could be called intrinsic or internal symmetries since they do not have a direct geometrical action. As a concrete example we could think of charge-conjugation or isospin rotations. Thus we consider a symmetry which maps the observables of each region onto themselves:

$$
G B(R) G^{-1}=B(R) .
$$

The analogous condition in field theory would be that $G \phi_{\alpha}(x) G^{-1}$ is a local function of the fields $\phi$ at the point $x$. However this concept of local function is not very well defined, and a more natural condition would be

$$
\left[G \phi_{\alpha}(x) G^{-1}, \phi_{\mu}(y)\right]=0 \text { for }(x-y)^{2}<0 .
$$

The analogous condition to (4) in the case of local observables would then be

$$
\left[G B\left(R_{1}\right) G^{-1}, B\left(R_{2}\right)\right]=0 \quad \text { for } \quad R_{2} \subset R_{1}^{\prime} .
$$

In addition to condition (4) or $\left(3^{\prime}\right)$ we add the condition that $G$ leave the vacuum invariant:

$$
G|\operatorname{vac}\rangle=|\operatorname{vac}\rangle
$$

Conditions (4), ( $\left.3^{\prime}\right)$, and (5) constitute the basic conditions on the local internal symmetry $G$ and will be the starting point for the analysis of the general form for such a symmetry. No assumptions will be made concerning the nature of the group of internal symmetries or the group of the combined internal and Poincare transformations. In particular it is not assumed that the symmetries form a Lie group. The analysis will be carried out for a single symmetry operator $G$. 
It is also pointed out that although we have discussed only observables and commuting fields, in general one deals with a local theory which may contain operators which anticommute for space-like separation. For simplicity of discussion it will be assumed that the observables describe the entire local structure and only commuting fields will be discussed. However the same results can be obtained by assuming $G$ has a local action on a field algebra generated by Bose and Fermi type elements.

The main results will be to show that $G$ must commute with the Poincaré group and to determine the action of $G$ on the asymptotic particles and fields.

\section{The Translation Invariance of $G$}

We first consider the translation invariance of $G$ and review results previously obtained [3]. In the Hilbert space of physical states there is a unitary representation of the translations $U(x)$ which act in a local manner on the observables:

$$
U(x) B(R) U(x)^{-1}=B(R+x)
$$

where $R+x$ denotes the region obtained by translating each point in $R$ by the amount $x$. The generator of the translations, the four-momentum $P$, lies in the forward cone:

$$
P^{2} \geqq 0 \quad P^{4} \geqq 0
$$

where $P^{4}$ denotes the time component of $P$ and the metric is $P^{2}=\left(P^{4}\right)^{2}-(\boldsymbol{p})^{2}$. The condition (6) is the spectral condition.

Consider the operator

$$
G(y)=U(y) G U(y)^{-1} .
$$

If $G$ commutes with translations then $G(y)$ is independent of $y$. In any case it can be seen that since $G$ is an internal symmetry then $G(y)$ is also an internal symmetry. One then considers the function

$$
F(y, x)=\left\langle\operatorname{vac}\left|\left[G(y) b_{1} G(y)^{-1}, b_{2}(x)\right]\right| \operatorname{vac}\right\rangle
$$

where $b_{1}$ and $b_{2}$ are arbitrary operators from some region $R$. By combining locality, which gives conditions on $F(y, x)$ in coordinate space $x$, with the spectral condition, which gives conditions on the Fourier transform $\tilde{F}(y, p)$ in momentum space $p$, one can show that $F(y, x)$ is actually independent of $y$. From this it can be shown that $G(y)$ is independent of $y$ and thus that $G$ commutes with translations. Details can be found in Ref. [3]. 
This result means that there is no mass-splitting for an internal symmetry group. All particles in a multiplet have the same mass. This result also shows, for example, that there does not exist a unitary operator $G$ such that the action of $G$ on a set of fields $\phi_{\alpha}(x)$ is given by

$$
G \phi_{\alpha}(x) G^{-1}=\mathscr{G}_{\alpha \mu}(x) \phi_{\mu}(x)
$$

where $\mathscr{G}_{\alpha \mu}$ depends on $x$.

We now go on to study the Lorentz transformation properties of $G$, and thus we consider the unitary representation of the Lorentz group $U(\Lambda)$ which acts in the correct local manner on the observables:

$$
U(\Lambda) B(R) U(\Lambda)^{-1}=B\left(R_{\Lambda}\right)
$$

where $R_{A}$ is the set of points obtained by performing a Lorentz transformation $\Lambda$ on each point in $R$. The Lorentz transformations together with the translations give a representation of the Poincare group. It will be shown within a field theoretical framework that $G$ must commute with the Lorentz group. Thus in particular we conclude that $G$ must commute with rotations, and thus all particles in a multiplet must have the same spin, thereby excluding groups such as $S U(6)$ as internal symmetry groups.

\section{Asymptotic States}

In order to determine the action of the symmetry $G$ on the particles in the theory, we must have a connection between the local algebras or interacting fields and the asymptotic free particle states. This connection is given by the Haag-Ruelle construction, or alternatively by the LSZ asymptotic conditions. The Haag-Ruelle theory has not yet been extended to include massless particles, and primarily for this reason we add the condition that there are no zero mass particles in the theory. The question of the effect of this condition on the general results can only be answered after a more complete theory of massless particles is developed.

Assuming no zero mass particles, the Haag-Ruelle asymptotic states can be constructed in the usual manner [5], and assuming a complete particle interpretation to the theory, these asymptotic states give rise to the entire Hilbert space of physical states. It will be assumed that there are a finite number of particles at each mass value. Infinitely degenerate multiplets are considered in Section 9.

From the condition that $G$ commutes with translations it follows that $G$ must map states of each fixed momentum $p$ onto themselves, and thus $G$ maps one-particle states onto one-particle states. To determine the action of $G$ on the many-particle asymptotic states, we review briefly the construction of the asymptotic states, and show that $G$ acts multiplica- 
tively on the $n$-particle states. That is, $G$ acts on an $n$-particle state as the tensor product of its action on the one-particle states.

The asymptotic states are constructed as follows. Let $Q$ be a local operator which has non-zero matrix elements between the vacuum and a one-particle state of mass $m$, and let $Q(x)$ be the translated operator $U(x) Q U(x)^{-1}$. Then define

$$
q=\int d^{4}(x) Q(x) h(x)
$$

where $h(x)$ is an infinitely differentiable function which falls to zero rapidly as $x$ approaches infinity, and has support in momentum space confined to a neighborhood of the mass-shell $p^{2}=m^{2}$. Then when $q$ acts on the vacuum it will create a one-particle state of mass $m$. In fact a set of operators $q_{i}$ can be constructed such that $q_{i}$ creates a particle of type $i$ from the vacuum:

$$
q_{i}|\operatorname{vac}\rangle=\int d^{3}(\boldsymbol{p}) \phi_{i}(\boldsymbol{p})|\boldsymbol{p} i\rangle
$$

and such that $\phi_{i}(\boldsymbol{p})$ is infinitely often differentiable and non-vanishing [5]. Here $i$ denotes the spin and any other quantum numbers describing the particles of mass $m$.

Then the operator

$$
q(f, t)=\sum_{i} \int d^{3}(\boldsymbol{x}) g_{i}(\boldsymbol{x}, t) q_{i}(\boldsymbol{x}, t)
$$

when acting on the vacuum creates a one-particle state with wavefunction $f_{i}(\boldsymbol{p})$ if $g_{i}(\boldsymbol{x}, t)$ is selected such that

That is

$$
g_{i}(\boldsymbol{x}, t)=\int d^{3}(\boldsymbol{p}) \frac{f_{i}(\boldsymbol{p})}{\phi_{i}(\boldsymbol{p})} \exp \left[i\left(\boldsymbol{p} \cdot \boldsymbol{x}-\sqrt{p^{2}+m^{2}} t\right)\right]
$$

independent of $t$.

$$
q(f, t)|\operatorname{vac}\rangle=|\mathrm{f}\rangle
$$

The operator $q(x)$ is not a local operator since $Q(x)$ in expression (7) is integrated over all space-time. However $q(x)$ is an almost local operator $^{1}$ since $h(x)$ falls to zero rapidly for large $x$. This can be used to show [5] that $q\left(f_{1}, t\right) \ldots q\left(f_{n}, t\right)|\mathrm{vac}\rangle$ has a strong limit as $t \rightarrow \infty$ :

$$
\left|f_{1} \ldots f_{n}\right\rangle=\lim _{t \rightarrow \infty} q\left(f_{1}, t\right) \ldots q\left(f_{n}, t\right)|\mathrm{vac}\rangle .
$$

Moreover, this limit is not sensitive to the choice of $q(x)$ as stated in the following theorem [6]:

If $q_{2}(x)$ is almost local with respect to $q_{1}(x)$, and if

$$
q_{1}(f, t) \equiv \int d^{3}(\boldsymbol{x}) q_{1}(\boldsymbol{x}, t) g_{1}(\boldsymbol{x}, t) \quad \text { and } \quad q_{2}(f, t) \equiv \int d^{3}(\boldsymbol{x}) q_{2}(\boldsymbol{x}, t) g_{2}(\boldsymbol{x}, t)
$$

${ }_{1}$ Two operators $q_{1}$ and $q_{2}$ are said to be almost local with respect to each other if the commutator $\left[q_{1}, q_{2}(\boldsymbol{x})\right]$ goes to zero faster than any inverse power of $|\boldsymbol{x}|$ as $\boldsymbol{x} \rightarrow \infty$. 
create the same one-particle state from the vacuum

$$
q_{1}(f, t)|\mathrm{vac}\rangle=q_{2}(f, t)|\mathrm{vac}\rangle=|f\rangle
$$

then $q_{1}(f, t)$ and $q_{2}(f, t)$ can be used interchangeably in the construction of the states $\left|f_{1} \ldots f_{n}\right\rangle$.

The state $\left|f_{1} \ldots f_{n}\right\rangle$ can be interpreted as an asymptotic $n$-particle state because the Poincare transformations act multiplicatively on these states:

$$
U(\lambda)\left|f_{1} \ldots f_{n}\right\rangle=\left|f_{1 \lambda} \ldots f_{n \lambda}\right\rangle
$$

where $f_{\lambda}$ is determined by the action of $U(\lambda)$ on the one-particle states $|f\rangle$ :

$$
U(\lambda)|f\rangle=\left|f_{\lambda}\right\rangle .
$$

This can be shown in a manner analogous to the discussion given below of the action of the internal symmetry $G$ on the asymptotic states.

One can then introduce creation-destruction operators such that

$$
\left|f_{1} \ldots f_{n}\right\rangle=N a^{\dagger}\left(f_{1}\right) \ldots a^{\dagger}\left(f_{n}\right)|\mathrm{vac}\rangle
$$

where $N$ is an appropriate normalization constant.

Consider now the action of the symmetry $G$ on the asymptotic states. Since $G$ maps one-particle states onto themselves we have

$$
G|f\rangle=\left|f_{G}\right\rangle
$$

where $|f\rangle$ and $\left|f_{G}\right\rangle$ are one-particle states. Also, since $G$ acts locally on the observables as given by $\left(3^{\prime}\right)$ it follows from (7) that $G q(x) G^{-1}$ is almost local with respect to $q(y)$. From the above mentioned theorem, one concludes that $G q(f, t) G^{-1}$ can be used to construct the asymptotic states with wave-function $f_{G}$, and thus

$$
\begin{aligned}
G\left|f_{1} \ldots f_{n}\right\rangle & =G \lim _{t \rightarrow \infty} q\left(f_{1}, t\right) \ldots q\left(f_{n}, t\right)|\mathrm{vac}\rangle \\
& =\lim _{t \rightarrow \infty} G q\left(f_{1}, t\right) G^{-1} \ldots G q\left(f_{n}, t\right) G^{-1}|\mathrm{vac}\rangle=\left|f_{1 G} \ldots f_{n G}\right\rangle .
\end{aligned}
$$

Thus $G$ acts multiplicatively on the $n$-particle states. This important property holds for any local symmetry operator. From this we can immediately conclude several results. Since a one-particle "in"-state is the same as a one-particle "out"-state, it is seen that $G$ has the same action on an $n$-particle "in"-state as on an $n$-particle "out"-state, and thus $G$ commutes with the $S$-matrix. Also, if $G(\lambda)$ is a one-parameter internal symmetry group generated by a charge $Q$

$$
G(\lambda)=e^{i \lambda Q}
$$

then $Q$ is an additive quantity which is conserved. 
The most general form for the action of $G$ on a one-particle state is

$$
G|\boldsymbol{p} i\rangle=g_{i j}(\boldsymbol{p})|\boldsymbol{p} j\rangle
$$

where $g_{i j}$ is a unitary matrix for each $\boldsymbol{p}$, and we sum over the repeated index. From the multiplicative character of $G$ it follows that

$$
G a_{i}^{\dagger}(\boldsymbol{p}) G^{-1}=g_{i j}(\boldsymbol{p}) a_{j}^{\dagger}(\boldsymbol{p})
$$

i.e. $G$ acts linearly on the asymptotic creation operators, a direct result of the local character of $G$ [7]. It has thus been shown that although $G$ may have a very non-linear action on the interacting fields, $G$ acts linearly on the asymptotic fields. We will next show that $G$ acts locally on the asymptotic fields.

Before proceeding we make the following remark. In the above discussion in which it was concluded that $G$ acts multiplicatively on the asymptotic states, it was essential that $G$ act in a local manner on the observables $B(R)$. For consider a unitary operator which commutes with the Poincare group, the $S$-matrix, and define

$$
\tilde{B}(R)=S B(R) S^{-1} .
$$

Clearly $\tilde{B}(R)$ form a local system with the same transformation properties under the Poincaré group, but $\tilde{B}(R)$ is not relatively local to $B(R)$. The asymptotic "out"-states associated with $\tilde{B}(R)$ are just the asymptotic "in"-states associated with $B(R)$. It is only when the condition of relative locality is imposed that one obtains the same asymptotic states.

\section{Asymptotic Locality and the Action of $G$ on the Asymptotic Fields}

We introduce the notion of asymptotic locality, which is the property that the local structure associated with a system of operators is preserved in the transition from interacting fields to asymptotic fields.

Given a local operator $Q \in B(R)$ and its translates $Q(x)$, one can define an operator $\hat{Q}(x)$ which is linear in creation, destruction operators, by the expression

$$
\hat{Q}(x)=\sum_{i} \int d^{3}(\boldsymbol{p})\langle\boldsymbol{p} i|Q(x)| \operatorname{vac}\rangle a_{i}^{\dagger}(\boldsymbol{p})+\langle\operatorname{vac}|Q(x)| p i\rangle a_{i}(\boldsymbol{p})
$$

where the summation includes only those particle states having a single mass value $m$. In fact it was shown in reference [5] that $\hat{Q}(x)$ is the asymptotic operator associated with $Q(x)$, in the sense that in the limit $t \rightarrow \infty$ the matrix elements of $Q(x)$ approach those of $\hat{Q}(x)$ when smeared with suitable test functions.

It will now be shown that the mapping $Q(x) \rightarrow \hat{Q}(x)$ preserves the local structure associated with $Q(x)$. In particular suppose $Q_{1}$ and $Q_{2}$ 
are contained in $B\left(R_{1}\right)$, where $R_{1}$ is a double cone centered about the origin with base $|\boldsymbol{x}|<r$. Then $Q_{1}$ commutes with $Q_{2}(x)$ whenever $x \in R_{2}^{\prime}$, where $R_{2}$ is the double cone centered about the origin with base $|x|<2 r$. We now show that $\hat{Q}_{1}$ commutes with $\hat{Q}_{2}(x)$ for $x$ in the same region $R_{2}^{\prime}$. The commutator of $\hat{Q}_{1}$ and $\hat{Q}_{2}(x)$ is given by

$$
\begin{aligned}
& {\left[\hat{Q}_{1}, \hat{Q}_{2}(x)\right]} \\
& =\sum_{i} \int d^{3}(\boldsymbol{p})\left\langle\operatorname{vac}\left|Q_{1}\right| \boldsymbol{p} i\right\rangle\left\langle\boldsymbol{p} i\left|Q_{2}(x)\right| \operatorname{vac}\right\rangle-\left\langle\operatorname{vac}\left|Q_{2}(x)\right| \boldsymbol{p} i\right\rangle\left\langle\boldsymbol{p} i\left|Q_{1}\right| \mathrm{vac}\right\rangle \\
& =\left\langle\operatorname{vac}\left|\left[Q_{1}, Q_{2}(x)\right]\right| \operatorname{vac}\right\rangle_{m}
\end{aligned}
$$

where $\left\langle\operatorname{vac}\left|\left[Q_{1}, Q_{2}(x)\right]\right| v a c\right\rangle_{m}$ denotes the contribution of the oneparticle states of mass $m$ to the vacuum expectation value of the commutator. Defining

$$
F(x)=\left\langle\operatorname{vac}\left|\left[Q_{1}, Q_{2}(x)\right]\right| \mathrm{vac}\right\rangle
$$

we must show that the part of $F(x)$ containing a delta-function singularity $\delta\left(p^{2}-m^{2}\right)$ in momentum space must vanish in the same region $R_{2}^{\prime}$ as $F(x)$ itself. The argument is based on a Jost-Lehmann-Dyson representation for $F(x)$ which is derived as follows.

Since the support of the Fourier transform $\tilde{F}(p)$ in momentum space is contained in the region $p^{2} \geqq 0, F(x)$ is the boundary value for $s=0$ of a tempered distribution in five variables $F(x, s)$ which satisfies the wave equation [8]

$$
\left(\partial_{t}^{2}-\nabla^{2}-\partial_{s}^{2}\right) F(x, s)=0 .
$$

The condition that $F(x, 0)=0$ for $x \in R_{2}^{\prime}$ implies that the boundary values of $F(x, s)$ on the plane $t=0$ must vanish except in the "strip" $|x| \leqq 2 r$, all $s$. This follows from uniqueness theorems for the wave equation [9]. If $F(x, s)$ is now expressed in terms of its boundary values on the plane $t=0$ we obtain

$$
\begin{aligned}
F(x, s)= & \int d^{3}\left(\boldsymbol{x}^{\prime}\right) d s^{\prime}\left[\Delta_{0}^{5}\left(t, \boldsymbol{x}-\boldsymbol{x}^{\prime}, s-s^{\prime}\right) f_{1}\left(\boldsymbol{x}^{\prime}, s^{\prime}\right)\right. \\
& \left.+\partial_{t} \Delta_{0}^{5}\left(t, \boldsymbol{x}-\boldsymbol{x}^{\prime}, s-s^{\prime}\right) f_{2}\left(\boldsymbol{x}^{\prime}, s^{\prime}\right)\right]
\end{aligned}
$$

where $\Delta_{0}^{5}$ is the propagator for the five-dimensional wave equation and $f_{1,2}$ have supports contained in the region $|\boldsymbol{x}| \leqq 2 r$.

Expressing $\Delta_{0}^{5}(x, s)$ in the form

$$
\begin{aligned}
\Delta_{0}^{5}(x, s) & =\frac{i}{(2 \pi)^{4}} \int d^{4}(p) d p_{s} \varepsilon\left(p^{4}\right) \delta\left(p^{2}-p_{s}^{2}\right) \exp i\left(p \cdot x-p_{s} s\right) \\
& =\frac{1}{(2 \pi)} \int_{-\infty}^{\infty} d p_{s} \Delta_{0}\left(x, p_{s}^{2}\right) \exp \left(-i p_{s} s\right)
\end{aligned}
$$


where $\Delta_{0}(x)$ is the usual commutator function in four dimensions, and setting $s=0$ in Eq. (9), the following representation for $F(x)$ is obtained:

$$
\begin{aligned}
F(x)= & \int_{-\infty}^{\infty} d p_{s} \int d^{3}\left(\boldsymbol{x}^{\prime}\right)\left[\Delta_{0}\left(t, \boldsymbol{x}-\boldsymbol{x}^{\prime}, p_{s}^{2}\right) \hat{f}_{1}\left(\boldsymbol{x}^{\prime}, p_{s}\right)\right. \\
& \left.+\partial_{t} \Delta_{0}\left(t, \boldsymbol{x}-\boldsymbol{x}^{\prime}, p_{s}^{2}\right) \hat{f}_{2}\left(\boldsymbol{x}^{\prime}, p_{s}\right)\right]
\end{aligned}
$$

where $\hat{f}\left(\boldsymbol{x}^{\prime}, p_{s}\right)$ denotes the Fourier transform of $f\left(\boldsymbol{x}^{\prime}, s\right)$ with respect to $s$. Now it can be seen that since $\hat{f}_{1,2}\left(\boldsymbol{x}, p_{s}\right)$ vanish for $|\boldsymbol{x}|>2 r$ and $\Delta_{0}(x)$ vanishes for space-like $x$, the contribution to $F(x)$ from each $p^{2}\left(=p_{s}^{2}\right)$ value will vanish for $x \in R_{2}^{\prime}$. In particular if $\hat{f}_{1,2}\left(\boldsymbol{x}, p_{s}\right)$ has a delta function singularity for $p_{s}^{2}=m^{2}$, the corresponding contribution to $F(x)$ will vanish in the same region as $F(x)$ itself. This completes the demonstration that the local structure is preserved in the mapping to asymptotic operators. This property will be called "asymptotic locality".

In the case of field theory, we conclude that if

then

$$
\left[\phi_{\alpha}(x), \phi_{\mu}(y)\right]=0 \text { for }(x-y)^{2}<0
$$

$$
\left[\hat{\phi}_{\alpha}(x), \hat{\phi}_{\mu}(y)\right]=0 \text { for }(x-y)^{2}<0
$$

where $\hat{\phi}_{\alpha}$ is the asymptotic field associated with $\phi_{\alpha}$ and is given by Eq. (8). It is thus seen in a simple way that if a set of fields belong to a single Borchers' class, then their asymptotic fields will also belong to a single Borchers' class.

We are now in a position to investigate the action of the symmetry $G$ on the asymptotic operators $\hat{Q}(x)$. We first observe that since

$$
G a_{i}^{\dagger}(\boldsymbol{p}) G^{-1}=g_{i j}(\boldsymbol{p}) a_{j}^{\dagger}(\boldsymbol{p})
$$

it can be concluded that

$$
\begin{aligned}
G \hat{Q}(x) & G^{-1} \\
& =\sum_{i} \int d^{3}(\boldsymbol{p})\langle\boldsymbol{p} i|Q(x)| \operatorname{vac}\rangle G a_{i}^{\dagger}(\boldsymbol{p}) G^{-1}+\langle\operatorname{vac}|Q(x)| \boldsymbol{p} i\rangle G a_{i}(\boldsymbol{p}) G^{-1} \\
& =\sum_{i} \int d^{3}(\boldsymbol{p})\langle\boldsymbol{p} i|G Q(x)| \operatorname{vac}\rangle a_{i}^{\dagger}(\boldsymbol{p})+\left\langle\operatorname{vac}\left|Q(x) G^{-1}\right| \boldsymbol{p} i\right\rangle a_{i}(\boldsymbol{p}) \\
& =\widehat{G Q(x) G^{-1}}
\end{aligned}
$$

where the last symbol denotes the asymptotic operator associated with $G Q(x) G^{-1}$. In other words, the asymptotic operator associated with $G Q(x) G^{-1}$ is given by the action of $G$ on the asymptotic operator $\hat{Q}(x)$. It then follows, since $G Q(x) G^{-1}$ is local relative to $Q(y)$, that $G \hat{Q}(x) G^{-1}$ is local relative to $\hat{Q}(y)$. That is, $G$ acts locally on the asymptotic operators if it acts locally on the observables $B(R)$. 
In the case of fields, one would conclude from

that

$$
\left[G \phi_{\alpha}(x) G^{-1}, \phi_{\mu}(y)\right]=0 \text { for }(x-y)^{2}<0
$$

$$
\left[G \hat{\phi}_{\alpha}(x) G^{-1}, \hat{\phi}_{\mu}(y)\right]=0 \text { for }(x-y)^{2}<0
$$

Thus it has been shown that $G$ acts linearly and locally on the asymptotic fields. This condition allows a great simplification in the analysis of the general structure of $G$. Up to this point the discussion has been very general, applying to any local theory. We will from this point on restrict our attention to field theory, and in particular, to an interpolating field theory. That is, we assume that for each particle in the theory there exists a field with non-zero matrix elements between the vacuum and oneparticle states. This allows a simplified discussion of the action of the symmetry $G$ on the asymptotic fields, and will lead to the conclusion that $G$ must commute with the Lorentz group. The analysis is carried out in the next few sections. It should be possible to make a similar analysis without the assumption of interpolating fields, provided there are a finite number of particles at each mass, although a more detailed analysis of the operators $\hat{Q}(x)$ would have to be given. In this connection, see also Section 9.

\section{The Choice of Asymptotic Fields}

We consider now an interpolating field theory: a system of finite component fields $\phi_{\alpha}(x)$ such that there exists a field operator with nonzero matrix elements between the vacuum and each particle state. The asymptotic fields for a given mass value are given by Eq. (8) of the preceding section:

$$
\hat{\phi}_{\alpha}(x)=\sum_{i} \int d^{3}(\boldsymbol{p})\left\langle\boldsymbol{p} i\left|\phi_{\alpha}(x)\right| \operatorname{vac}\right\rangle a_{i}^{\dagger}(\boldsymbol{p})+\left\langle\operatorname{vac}\left|\phi_{\alpha}(x)\right| \boldsymbol{p} i\right\rangle a_{i}(\boldsymbol{p}) .
$$

From the results of the previous sections, the symmetry $G$ acts linearly and locally on these asymptotic fields. It is convenient in the analysis of the structure of $G$ to make a particular choice of asymptotic fields. We briefly review the discussion of J. Schwartz [10] concerning the reduction of a system of free fields to a certain canonical form.

Given a system of free fields, all having the same mass $p^{2}=m^{2}$, one can form, by differentiation, a system of fields transforming according to the $\left(0, s_{i}\right)$ representation of the Lorentz group, for some collection of $s_{i}$. That is, each field can be put in the form $\phi_{\alpha_{1} \ldots \alpha_{m}}(x)$ where $\phi$ is symmetric in $\alpha$ and transforms as

$$
U(\lambda)^{-1} \phi_{\alpha_{1} \ldots \alpha_{m}}(x) U(\lambda)=u_{\alpha_{1} \alpha_{1}^{\prime}} \ldots u_{\alpha_{m} \alpha_{m}^{\prime}} \phi_{\alpha_{1}^{\prime} \ldots \alpha_{m}^{\prime}}\left(M(\lambda)^{-1} x\right)
$$


where $u_{\alpha \alpha^{\prime}}$ is the representation of the (covering group of the) Lorentz group given by unimodular two-by-two matrices.

In addition the fields can be chosen to satisfy the "reality condition"

where

$$
\phi_{\alpha_{1} \ldots \alpha_{m}}^{\dagger}(x)=\partial_{\alpha_{1} \alpha_{1}^{\prime}} \ldots \partial_{\alpha_{m} \alpha_{m}^{\prime}} \phi_{\alpha_{1}^{\prime} \ldots \alpha_{m}^{\prime}}(x)
$$

$$
\begin{aligned}
\partial_{\alpha \alpha^{\prime}} & =\sum_{n \mu} \sigma_{\alpha \mu}^{n} \varepsilon_{\mu \alpha^{\prime}} \partial_{n}, \\
\varepsilon_{\mu \alpha} & =\left(\begin{array}{rr}
0 & -1 \\
1 & 0
\end{array}\right),
\end{aligned}
$$

$\sigma_{\alpha \mu}^{4}=\delta_{\alpha \mu}$, and $\sigma^{1,2,3}$ are Pauli matrices.

The commutator of fields transforming according to a single irreducible representation of the Lorentz group is given by

$$
\left[\phi_{\alpha_{1} \ldots \alpha_{m}}(x), \phi_{\alpha_{1}^{\prime} \ldots \alpha_{m}^{\prime}}(y)\right]=i^{m+1} \int_{\alpha} \varepsilon_{\alpha_{1} \alpha_{1}^{\prime}} \ldots \varepsilon_{\alpha_{m} \alpha_{m}^{\prime}} \Delta_{0}(x-y)
$$

where $S$ denotes symmetrization with respect to $\alpha$. The commutator of fields belonging to different irreducible representations of the Lorentz group is identically zero.

The interpretation of these fields is the following. The fields can be Fourier analyzed in terms of particle creation-destruction operators. The reality condition then implies that the destruction operators within a single field are associated with the same particle as the creation operators. The number of components of each field is equal to the number of spinstates of the particle. The vanishing of the commutator between different fields expresses the independence of the creation operators associated with the different fields. Thus there is a one-to-one correspondence between fields and particles. Each particle of spin $s$ is associated with a single field transforming according to the $(0, s)$ representation of the Lorentz group. For further discussion of these fields in terms of creationdestruction operators, see Weinberg [11]. The original system of fields can be obtained by again differentiating the $(0, s)$ fields. Thus this canonical set of fields is completely equivalent to the original fields.

Denoting for convenience

$$
\psi_{\alpha_{1} \ldots \alpha_{m}}(x)=(-i)^{m+1} \varepsilon_{\alpha_{1} \alpha_{1}^{\prime}} \ldots \varepsilon_{\alpha_{m} \alpha_{m}^{\prime}} \phi_{\alpha_{1}^{\prime} \ldots \alpha_{m}^{\prime}}(x)
$$

it follows from (11) that

$$
\left[\phi_{\alpha_{1} \ldots \alpha_{m}}(\boldsymbol{x}, 0), \psi_{t \mu_{1} \ldots \mu_{m}}(\boldsymbol{y}, 0)\right]=\underset{\alpha}{S} \delta_{\alpha_{1} \mu_{1}} \ldots \delta_{\alpha_{m} \mu_{m}} \delta^{3}(\boldsymbol{x}-\boldsymbol{y})
$$

where $\psi_{t \mu_{1} \ldots \mu_{m}}$ denotes the time derivative of $\psi_{\mu_{1} \ldots \mu_{m}}$. 
We can now show explicitly that if $G \phi_{\alpha_{1} \ldots \alpha_{m}}(x) G^{-1}$ commutes with $\phi_{\alpha_{1} \ldots \alpha_{k}}(y)$ for $(x-y)^{2}<0$, then $G \phi G^{-1}$ is a polynomial in derivatives acting on the fields $\phi$.

Consider first an arbitrary operator $Q$ which is linear in creationdestruction operators. $Q$ can be expressed in terms of the fields and their time-derivatives on the plane $t=0$ :

$$
Q=\phi_{\alpha_{1} \ldots \alpha_{m}}\left(f^{\alpha_{1} \ldots \alpha_{m}}, 0\right)+\phi_{t \alpha_{1} \ldots \alpha_{m}}\left(g^{\alpha_{1} \ldots \alpha_{m}}, 0\right)
$$

where we sum over $\alpha$ and over the different $m$-values. Then from Eq. (12), $f$ and $g$ are given explicitly by

$$
\begin{aligned}
f^{\alpha_{1} \ldots \alpha_{m}}(\boldsymbol{x}) & =\left[Q, \psi_{t \alpha_{1} \ldots \alpha_{m}}(\boldsymbol{x}, 0)\right], \\
g^{\alpha_{1} \ldots \alpha_{m}}(\boldsymbol{x}) & =-\left[Q, \psi_{\alpha_{1} \ldots \alpha_{m}}(\boldsymbol{x}, 0)\right] .
\end{aligned}
$$

Therefore $Q(x)$ is given by

$$
\begin{aligned}
Q(x)= & \int d^{3}(\boldsymbol{y})-\left[Q(x), \psi_{\alpha_{1} \ldots \alpha_{m}}(\boldsymbol{y}, 0)\right] \phi_{t \alpha_{1} \ldots \alpha_{m}}(\boldsymbol{y}, 0) \\
& +\left[Q(x), \psi_{t \alpha_{1} \ldots \alpha_{m}}(\boldsymbol{y}, 0)\right] \phi_{\alpha_{1} \ldots \alpha_{m}}(\boldsymbol{y}, 0) \\
= & -\int d^{3}(\boldsymbol{y})\left[Q(x), \psi_{\alpha_{1} \ldots \alpha_{m}}(\boldsymbol{y}, 0)\right]{\overleftrightarrow{\partial_{t y}}}_{t_{\alpha_{1}} \ldots \alpha_{m}}(\boldsymbol{y}, 0) .
\end{aligned}
$$

When $Q(x)$ is relatively local to the fields $\phi(y)$, the commutator of $Q(x)$ with $\psi(y)$ has the form

$$
\left[Q(x), \psi_{\alpha_{1} \ldots \alpha_{m}}(y)\right]=K_{\alpha_{1} \ldots \alpha_{m}}\left(-i \partial_{x}\right) \Delta_{0}(x-y)
$$

where $K(-i \partial)$ is a polynomial in derivatives acting on $\Delta_{0}$, and therefore

$$
\begin{aligned}
Q(x) & =-K_{\alpha_{1} \ldots \alpha_{m}}\left(-i \partial_{x}\right) \int d^{3}(\boldsymbol{y}) \Delta_{0}(t, \boldsymbol{x}-\boldsymbol{y}) \overleftrightarrow{\partial}_{t_{y}} \phi_{\alpha_{1} \ldots \alpha_{m}}(\boldsymbol{y}, 0) \\
& =K_{\alpha_{1} \ldots \alpha_{m}}\left(-i \partial_{x}\right) \phi_{\alpha_{1} \ldots \alpha_{m}}(x) .
\end{aligned}
$$

Thus $G \phi G^{-1}$ is a polynomial in derivatives acting on the fields $\phi$. From this we will show that $G$ must commute with the Lorentz group.

Before proceeding, we make the following remark. The above discussion has the following consequence. Let $\phi_{\alpha}(x)$ be a system of interacting fields, and denote by $P(R)$ the polynomial algebra of the fields smeared with test functions with support in the region $R$. Let $Q$ be an operator belonging to the polynomial algebra $P(R)$ with $R$ a double cone centered about the origin with base $|\boldsymbol{x}|<r$. Then since

$$
\left[Q, \phi_{\alpha}(x)\right]=0 \quad \text { for } \quad x \in R^{\prime}
$$

it follows that

$$
\left[\hat{Q}, \hat{\phi}_{\alpha}(x)\right]=0 \quad \text { for } \quad x \in R^{\prime}
$$


and therefore the functions $f(\boldsymbol{x})$ and $g(\boldsymbol{x})$ given by Eq. (13) will vanish for $|\boldsymbol{x}| \geqq r$, and thus $\hat{Q}$ will belong to the polynomial algebra of $\hat{\phi}_{\alpha}$ associated with the same double cone $R$. That is,

$$
Q \in P(R) \Rightarrow \hat{Q} \in \hat{P}(R)
$$

(where $\hat{Q}$ is actually linear in the fields $\hat{\phi}$ ).

\section{The Lorentz Invariance of Local Internal Symmetries}

We consider a system of free fields $\phi_{\alpha}(x)$ which are transformed under the unitary operator $G$ in the following manner:

$$
G \phi_{\alpha}(x) G^{-1}=K_{\alpha \mu}(-i \partial) \phi_{\mu}(x)
$$

where $K$ is a polynomial in derivatives acting on the fields $\phi$. Taking matrix elements of (14) between the vacuum and one-particle states, and using $G|\boldsymbol{p} i\rangle=g_{i j}(\boldsymbol{p})|\boldsymbol{p} j\rangle$, it follows that

$$
g_{j i}(\boldsymbol{p})\left\langle\boldsymbol{p} j\left|\phi_{\alpha}(0)\right| \mathrm{vac}\right\rangle=K_{\alpha \mu}(p)\left\langle\boldsymbol{p} i\left|\phi_{\mu}(0)\right| \mathrm{vac}\right\rangle .
$$

Using the fact that

$$
|\boldsymbol{p} i\rangle=\sqrt{\frac{m}{\omega(p)}} U(\boldsymbol{p})|\mathbf{0} i\rangle
$$

where $U(\boldsymbol{p})$ is a velocity transformation (boost, pure Lorentz transformation) which brings a rest state up to momentum $p$, Eq. (15) can be written in the form

$$
g_{j i}(\boldsymbol{p}) \Gamma_{\alpha \mu}(\boldsymbol{p})\left\langle\mathbf{0} j\left|\phi_{\mu}(0)\right| \mathrm{vac}\right\rangle=K_{\alpha \mu}(p) \Gamma_{\mu \lambda}(\boldsymbol{p})\left\langle\mathbf{0} i\left|\phi_{\lambda}(0)\right| \mathrm{vac}\right\rangle
$$

where $\Gamma_{\mu \lambda}(\boldsymbol{p})$ is the representative of the velocity transformation in the finite-dimensional representation of the Lorentz group according to which the fields $\phi_{\alpha}$ transform. In the particular representation for free fields discussed in the previous section, $\Gamma_{\mu \lambda}$ will be a direct sum of $(0, s)$ representations. Then

$$
\left\langle\mathbf{0} i\left|\phi_{\mu}(0)\right| \mathrm{vac}\right\rangle=\delta_{i \mu}
$$

since $\phi_{\mu}(0)|v a c\rangle$ transforms as a particle with spin $s$ and third component of angular momentum $\mu$ under rotations, and since each particle is associated with a single field. Thus it follows that

$$
\Gamma_{\alpha j}(\boldsymbol{p}) g_{j i}(\boldsymbol{p})=K_{\alpha \mu}(p) \Gamma_{\mu i}(\boldsymbol{p}) .
$$

(This could also have been obtained directly from Eq. (14) by expressing the fields $\phi$ in terms of creation operators and spinors.) 
We now have the following expression for the unitary matrix $g$ :

$$
g(\boldsymbol{p})=\Gamma(-\boldsymbol{p}) K(p) \Gamma(\boldsymbol{p}) .
$$

It will now be shown that the only way the left-hand side of (16) can be a bounded function of $\boldsymbol{p}$ is for $g$ to be independent of $\boldsymbol{p}$. From this it will easily follow that $G$ commutes with the Lorentz group.

To study the structure of $\Gamma(\boldsymbol{p})$, note that $\Gamma(0, s)$ is given by the symmetrized direct product of $\Gamma(0,1 / 2)$ matrices. Consider the form of $\Gamma_{(0,1 / 2)}(\boldsymbol{p})$ :

$$
\begin{aligned}
\Gamma(\boldsymbol{p}) & =e^{-i q \hat{p} \cdot \mathbf{K}}=e^{q \hat{p} \cdot \boldsymbol{J}}=e^{q / 2 \hat{p} \cdot \sigma} \\
& =\cosh (q / 2)+\sinh (q / 2) \hat{p} \cdot \sigma
\end{aligned}
$$

where $\omega / m=\cosh q, p / m=\sinh q$ and $\hat{p}$ is a unit vector in the direction of $\boldsymbol{p}$. Then $\Gamma(0,1 / 2)$ can be expressed in the form

$$
\Gamma(\boldsymbol{p})=\sqrt{\frac{m}{\omega+p}} \Gamma_{1}(p, \hat{p})=\sqrt{\frac{m}{\omega-p}} \Gamma_{2}(p, \hat{p})
$$

where

$$
\begin{aligned}
& \Gamma_{1}(p, \hat{p})=\frac{1}{2}\left[\left(\frac{\omega+p}{m}+1\right)+\left(\frac{\omega+p}{m}-1\right) \hat{p} \cdot \sigma\right], \\
& \Gamma_{2}(p, \hat{p})=\frac{1}{2}\left[\left(1+\frac{\omega-p}{m}\right)+\left(1-\frac{\omega-p}{m}\right) \hat{p} \cdot \sigma\right] .
\end{aligned}
$$

The matrices $\Gamma_{1}$ and $\Gamma_{2}$ are polynomials in $p$ and $\omega$ for fixed direction $\hat{p}$. If $s$ is integral, $\Gamma(0, s)$ contains an even number of $\Gamma(0,1 / 2)$ factors. Take half in the form with $\Gamma_{1}$ and the other half in the form with $\Gamma_{2}$. Then the factors involving $\sqrt{\omega+p}$ and $\sqrt{\omega-p}$ cancel, and we conclude that for integrals $s, \Gamma(0, s)$ is a polynomial in $p$ and $\omega$ for fixed $\hat{p}$. (See the discussion at the end of this section for the case when $s$ is half-integral.) It is now seen that Eq. (16) expresses $g(p)$ as a polynomial in $p$ and $\omega$ for fixed direction $\hat{p}$. Since $g(\boldsymbol{p})$ is bounded, and $p$ can go from plus to minus infinity with $\omega$ constrained on the mass shell, we conclude that in fact the polynomial must be independent of $p$ and $\omega$. Thus $g(\boldsymbol{p})$ can depend only on the direction $\hat{p}$. On the other hand, it is easily seen that the righthand side of (16) is continuous at the origin $p=0$ and thus $g$ is independent of $\hat{p}$. We have thus shown that $g(\boldsymbol{p})$ is independent of $\boldsymbol{p}$.

In order to conclude that $G$ must commute with the Lorentz group, consider the operator

$$
G(\lambda)=U(\lambda) G U(\lambda)^{-1}
$$


where $U(\lambda)$ is a Lorentz transformation. Since $G(\lambda)$ is a local internal symmetry if $G$ is, we can apply the above argument to $G(\lambda)$ to conclude that $g_{i j}(\lambda, \boldsymbol{p})$ defined by

$$
G(\lambda)|\boldsymbol{p} i\rangle=g_{i j}(\lambda, \boldsymbol{p})|\boldsymbol{p} j\rangle
$$

is independent of $\boldsymbol{p}$ for every Lorentz transformation $\lambda$. In particular, consider a velocity transformation $U(\boldsymbol{p})$, and apply $U(\boldsymbol{p}) G U(\boldsymbol{p})^{-1}$ to a rest state:

$$
U(\boldsymbol{p}) G U(\boldsymbol{p})^{-1}|\mathbf{0} i\rangle=U(\boldsymbol{p}) G|-\boldsymbol{p} i\rangle=U(\boldsymbol{p}) g_{i j}|-\boldsymbol{p} j\rangle=g_{i j}|\mathbf{0} j\rangle .
$$

This means that $G(\lambda)=G$ for all velocity transformations. However, since products of velocity transformations generate the entire Lorentz group (the product of two velocity transformations is a velocity transformation followed by a rotation) we conclude that $G(\lambda)=G$ for all Lorentz transformations, thus showing that $G$ commutes with the Lorentz group.

Because $G$ commutes with the Lorentz group, it is actually the case that no derivatives can occur in the expression (14) for $G \phi G^{-1}$. One way to see this is that the $n$th order derivatives of a $(0, s)$ field will transform, due to the Klein-Gordon equation, as $(n / 2, n / 2) \times(0, s)$, and the $(0, s)$ representation is not included in the decomposition of this product. Since $G \phi G^{-1}$ must transform under Lorentz transformations in the same way as $\phi$, it follows that no derivatives can occur in the expression.

We make two further comments concerning the above discussion. For simplicity we have been dealing with bose-type fields. We indicate here the modifications when fermi-fields are included. For half-integral values of $s, \Gamma(0, s)$ will contain an odd number of $\Gamma(0,1 / 2)$ factors. These $\Gamma(0,1 / 2)$ factors can be paired as in the above discussion, but there will be one factor left over. Thus $\Gamma(0, s)$ is of the form $\sqrt{\frac{m}{\omega+p}} \Gamma_{1}(0, s)$ where $\Gamma_{1}(0, s)$ is a polynomial in $p$ and $\omega$ for fixed $\hat{p}$. In the expression (16) for $g(\boldsymbol{p})$ the square-root factors will again cancel out, and we will again be left with a polynomial in $p$ and $\omega$, provided there are no cross-terms involving a representation with integral $s$ and a representation with halfintegral $s$. This cannot happen because $G$ cannot mix bose with fermi fields: $G \phi_{\alpha}(x) G^{-1}$ must satisfy the same commutation-anticommutation relations with $G \phi_{\mu}(y) G^{-1}$ that $\phi_{\alpha}(x)$ satisfies with $\phi_{\mu}(y)$, and this cannot happen if $G$ mixes bose with fermi fields.

The second comment concerns possible technical complications because we should actually only require (16) to hold almost everywhere. However it is actually the case that one can assume at the outset that $g_{i j}(\boldsymbol{p})$ is an infinitely differentiable function of $\boldsymbol{p}$. This follows from an 
argument based on that of Lemma 3 of Ref. [5]. From the local action of $G$ on the observables, it follows that $\left\langle\operatorname{vac}\left|q_{i}(\boldsymbol{x}) G q_{j} G^{-1}\right| \mathrm{vac}\right\rangle$ must decrease faster than any inverse power of $\boldsymbol{x}$ and therefore the Fourier transform $\phi_{i}(\boldsymbol{p}) \phi_{j}(\boldsymbol{p}) g_{j i}(\boldsymbol{p})$ is infinitely often differentiable in $\boldsymbol{p}$. Since $\phi_{i, j}$ are infinitely differentiable and non-vanishing it follows that $g_{i j}(p)$ is infinitely differentiable.

\section{The General Structure of $G$}

Using the above results, we can now describe the general action of a local internal symmetry on the asymptotic fields. Let $\phi_{\mu, \alpha_{1} \ldots \alpha_{j}}(x)$ be a system of free fields as described in Section 5. Under Lorentz transformations the field $\phi_{\mu, \alpha_{1} \ldots \alpha_{j}}$ transforms according to the $(0, j / 2)$ representation of the Lorentz group, and the index $\mu$ labels the different fields transforming according to the same $(0, j / 2)$ representation. Then the symmetry $G$ must act in the following manner on these asymptotic fields:

$$
G \phi_{\mu, \alpha_{1} \ldots \alpha_{j}}(x) G^{-1}=\mathscr{G}_{\mu \lambda} \phi_{\lambda, \alpha_{1} \ldots \alpha_{j}}(x) .
$$

From the reality condition, Eq. (10) of Section 5, it follows that

$$
\begin{aligned}
G \phi_{\mu, \alpha_{1} \ldots \alpha_{j}}^{\dagger}(x) G^{-1} & =\mathscr{G}_{\mu \lambda}^{*} \phi_{\lambda, \alpha_{1} \ldots \alpha_{j}}^{\dagger}(x) \\
& =G \partial_{\alpha_{1} \alpha_{1}^{\prime}} \ldots \partial_{\alpha_{j} \alpha_{j}^{\prime}} \phi_{\mu, \alpha_{1}^{\prime} \ldots \alpha_{j}^{\prime}}(x) G^{-1} \\
& =\mathscr{G}_{\mu \lambda} \phi_{\lambda, \alpha_{1} \ldots \alpha_{m}}^{\dagger}(x)
\end{aligned}
$$

and therefore $\mathscr{G}_{\mu \lambda}=\mathscr{\mathscr { G }}_{\mu \lambda}$

Thus $\mathscr{G}$ is a real matrix. This leads to Carruthers-type theorems [12] relating particles and antiparticles ${ }^{2}$. Thus for example, in the case of gauge transformations expressing the conservation of charge, there must be an antiparticle transforming according to $G a^{\dagger} G^{-1}=\exp (-i \theta) a^{\dagger}$ for each particle transforming according to $G a^{\dagger} G^{-1}=\exp (\mathrm{i} \theta) a^{\dagger}$, so that suitable linear combinations will give a real representation of the gauge group. Also, for isotopic spin, for particles transforming according to a half-integral representation, there must be antiparticles transforming according to the complex-conjugate representation.

We point out that since $G$ acts in the above simple way on the asymptotic fields, $G$ can be represented simply in terms of these fields. For instance, if the internal symmetry group is a one-parameter group generated by a charge, then the charge can be written as an integral of the fourth component of a four-vector current which is bilinear in the asymptotic fields.

\footnotetext{
${ }^{2}$ Because of the simple association of particles with fields, the same matrix $\mathscr{G}_{\mu \lambda}$ describes the action of $G$ on the particles.
}

12 Commun. math Phys., Vol. 17 
We mention a connection between the analysis given here and the work of Orzalesi, Sucher, and Woo [13]. Usually one associates a conserved four-vector current $j^{m}(x)$ with the generator $Q$ of a continuous symmetry by

$$
\begin{aligned}
Q & =\int d^{3}(\boldsymbol{x}) j^{4}(\boldsymbol{x}, t), \\
G(\lambda) & =e^{i \lambda Q} .
\end{aligned}
$$

Then $G$ will commute with the Poincare group because $j^{m}$ is a conserved current. Suppose we consider a conserved tensor

and define

$$
\partial_{m} T^{n m}(x)=0
$$

$$
Q^{n}=\int d^{3}(\boldsymbol{x}) T^{n 4}(\boldsymbol{x}, t) .
$$

Then $Q^{n}$ will not commute with the Lorentz group, and we conclude from the preceding analysis that $G(\lambda)=e^{i \lambda_{n} Q^{n}}$ cannot be an internal symmetry group. Thus it is not surprising that, as was shown in Ref. [13], under certain assumptions the operators $Q^{n}$ are actually proportional to the four-momentum $P^{n}$ and thus generate the translations.

\section{The Uniqueness of the Representation of the Poincaré Group}

In this section we consider the following question. Given a system of local observables, are the Poincaré transformations uniquely defined by their geometrical action on the observables? In other words, given an association of operators with regions $B(R)$, are the Poincare transformations uniquely determined by

$$
U(\lambda) B(R) U(\lambda)^{-1}=B\left(R_{\lambda}\right)
$$

where $R_{\lambda}$ is obtained by performing the Poincaré transformation on each space-time point in $R$.

If it were possible to have more than one representation of the Lorentz group then the spin of the particles described by the theory may not be uniquely determined. Under one choice of Lorentz transformation a particle may transform as a spin $1 / 2$ particle, and for another choice of Lorentz transformations the particle may transform like two spin zero particles. Or in terms of fields, could it be possible for a field to transform like a vector field for one choice of Lorentz transformation and like four scalar fields for another choice?

Suppose we have two representations of the Poincaré group:

$$
\begin{array}{ll}
U_{1}(\lambda) B(R) U_{1}(\lambda)^{-1}=B\left(R_{\lambda}\right) & U_{1}(\lambda)|\mathrm{vac}\rangle=|\mathrm{vac}\rangle, \\
U_{2}(\lambda) B(R) U_{2}(\lambda)^{-1}=B\left(R_{\lambda}\right) & U_{2}(\lambda)|\mathrm{vac}\rangle=|\mathrm{vac}\rangle .
\end{array}
$$


Then $G(\lambda)$ defined by

$$
G(\lambda)=U_{1}(\lambda)^{-1} U_{2}(\lambda)
$$

is a local internal symmetry for each $\lambda$. If we are dealing with a field theory with interpolating fields, we conclude from the results of the preceding sections, that $G(\lambda)$ commutes with $U_{1,2}\left(\lambda^{\prime}\right)$ for all $\lambda$ and $\lambda^{\prime}$. Then

$$
\begin{aligned}
G\left(\lambda_{1}\right) G\left(\lambda_{2}\right) & =G\left(\lambda_{1}\right) U_{1}\left(\lambda_{2}\right)^{-1} U_{2}\left(\lambda_{2}\right)=U_{1}\left(\lambda_{2}\right)^{-1} G\left(\lambda_{1}\right) U_{2}\left(\lambda_{2}\right) \\
& =U_{1}\left(\lambda_{1} \lambda_{2}\right)^{-1} U_{2}\left(\lambda_{1} \lambda_{2}\right)=G\left(\lambda_{1} \lambda_{2}\right) .
\end{aligned}
$$

Thus $G(\lambda)$ forms a representation of the Poincare group. Consider the action of $G(\lambda)$ on the one-particle states:

$$
G(\lambda)|\boldsymbol{p} i\rangle=g_{i j}(\lambda)|\boldsymbol{p} j\rangle .
$$

Since there are a finite number of particles at each mass, the matrices $g(\lambda)$ will form a unitary finite dimensional representation of the Poincaré group, the only one being the identity representation. Thus $g(\lambda)=I$ implying $G(\lambda)=I$ and $U_{2}(\lambda)=U_{1}(\lambda)$.

In order to see what may happen when one has infinitely degenerate particle multiplets we now investigate a simple example of an infinite component field and study the internal symmetries that it possesses.

\section{An Infinite Component Field}

Consider a system of creation-destruction operators

$$
a^{\dagger}(\boldsymbol{q}, \boldsymbol{p})
$$

labeled by two momenta $\boldsymbol{q}$ and $\boldsymbol{p}$. Only $\boldsymbol{p}$ is a true momentum in the sense that under translations

$$
U(z) a^{\dagger}(\boldsymbol{q}, \boldsymbol{p}) U(z)^{-1}=e^{i p \cdot z} a^{\dagger}(\boldsymbol{q}, \boldsymbol{p}) .
$$

(The three vectors $\boldsymbol{p}$ and $\boldsymbol{q}$ define four-vectors in the forward cone by $p^{2}=q^{2}=m^{2}$.) The creation operators satisfy the commutation relations

$$
\left[a\left(\boldsymbol{q}_{1}, \boldsymbol{p}_{1}\right), a^{\dagger}\left(\boldsymbol{q}_{2}, \boldsymbol{p}_{2}\right)\right]=\delta^{3}\left(\boldsymbol{q}_{1}-\boldsymbol{q}_{2}\right) \delta^{3}\left(\boldsymbol{p}_{1}-\boldsymbol{p}_{2}\right) .
$$

The delta function in $\boldsymbol{q}_{1}, \boldsymbol{q}_{2}$ indicates that the particle multiplets determined by $\boldsymbol{p}$ are really infinitely degenerate, the states $a^{\dagger}(\boldsymbol{q}, \boldsymbol{p})|\mathrm{vac}\rangle$ are orthogonal for different values of $\boldsymbol{q}$.

Under Lorentz transformations

$$
U(\lambda) a^{\dagger}(\boldsymbol{q}, \boldsymbol{p}) U(\lambda)^{-1}=\sqrt{\frac{\omega\left(\boldsymbol{q}_{\lambda}\right) \omega\left(\boldsymbol{p}_{\lambda}\right)}{\omega(\boldsymbol{q}) \omega(\boldsymbol{p})}} a^{\dagger}\left(\boldsymbol{q}_{\lambda}, \boldsymbol{p}_{\lambda}\right) .
$$


In order to determine what spins are contained in the representation of the Poincare group defined above, we look at the rest states $a^{\dagger}(\boldsymbol{q}, \mathbf{0})$. Under rotations

$$
U(R) a^{\dagger}(\boldsymbol{q}, \mathbf{0}) U(R)^{-1}=a^{\dagger}\left(\boldsymbol{q}_{R}, \mathbf{0}\right) .
$$

Thus $a^{\dagger}(\boldsymbol{q}, \boldsymbol{0})$ transforms like a state of momentum $\boldsymbol{q}$ and can be decomposed in the usual way into states of definite angular momentum:

$$
a^{\dagger}(j m, q, \mathbf{0}) .
$$

Thus for each value of the magnitude of $q$ all integral spins occur, and for each spin $j$ there are an infinity of states labeled by the magnitude of q. Thus we are dealing with an infinite degeneracy in spin as well as mass.

We can now form a local, infinite component free field by

$$
\phi(\boldsymbol{q}, x)=\int \frac{d^{3}(\boldsymbol{p})}{\sqrt{\omega(p)}}\left[a^{\dagger}(\boldsymbol{q}, \boldsymbol{p}) e^{i p \cdot x}+a(\boldsymbol{q}, \boldsymbol{p}) e^{-i p \cdot x}\right]
$$

which transforms as follows:

$$
\begin{aligned}
& U(z) \phi(\boldsymbol{q}, x) U(z)^{-1}=\phi(\boldsymbol{q}, x+z) \\
& U(\lambda) \phi(\boldsymbol{q}, x) U(\lambda)^{-1}=\sqrt{\omega\left(\boldsymbol{q}_{\lambda}\right) / \omega(\boldsymbol{q})} \phi\left(\boldsymbol{q}_{\lambda}, M(\lambda) x\right)
\end{aligned}
$$

and satisfies the commutation relations

$$
\left[\phi\left(\boldsymbol{q}_{1}, x_{1}\right), \phi\left(\boldsymbol{q}_{2}, x_{2}\right)\right]=\delta^{3}\left(\boldsymbol{q}_{1}-\boldsymbol{q}_{2}\right) \Delta_{0}\left(x_{1}-x_{2}\right) .
$$

(The field could also be defined by

$$
\psi(y, x)=\int \frac{d^{3}(\boldsymbol{q})}{\sqrt{\omega(q)}} \phi(\boldsymbol{q}, x) e^{i q \cdot y}
$$

which under Lorentz transformations transforms according to

$$
\left.U(\lambda) \psi(y, x) U(\lambda)^{-1}=\psi(M(\lambda) y, M(\lambda) x) .\right)
$$

Now it can be seen that $\phi$ has as an internal symmetry group the Lorentz group itself. An internal symmetry representation of the Lorentz group can be defined as follows. For each Lorentz transformation $\lambda$,

$$
\begin{aligned}
G(\lambda) a^{\dagger}(\boldsymbol{q}, \boldsymbol{p}) G(\lambda)^{-1} & =\sqrt{\omega\left(\boldsymbol{q}_{\lambda}\right) / \omega(\boldsymbol{q})} a^{\dagger}\left(\boldsymbol{q}_{\lambda}, \boldsymbol{p}\right) \\
G(\lambda)|\mathrm{vac}\rangle & =|\mathrm{vac}\rangle .
\end{aligned}
$$

The operators $G(\lambda)$ act locally on the field:

$$
G(\lambda) \phi(q, x) G(\lambda)^{-1}=\omega\left(q_{\lambda}\right) / \omega(q) \phi\left(q_{\lambda}, x\right) .
$$


It is clear that the internal symmetry group does not commute with the representation of the Lorentz group $U(\lambda)$.

In addition, the representation $U(\lambda)$ is not uniquely defined by its local action on the field $\phi(\boldsymbol{q}, x)$. We can define $U_{2}(\lambda)=G(\lambda)^{-1} U(\lambda)$ which will give a representation of the Lorentz group with the following properties:

$$
\begin{aligned}
U_{2}(\lambda) a^{\dagger}(\boldsymbol{q}, \boldsymbol{p}) & U_{2}(\lambda)^{-1}=\sqrt{\omega\left(\boldsymbol{p}_{\lambda}\right) / \omega(\boldsymbol{p})} a^{\dagger}\left(\boldsymbol{q}, \boldsymbol{p}_{\lambda}\right), \\
& U_{2}(\lambda) \phi(\boldsymbol{q}, x) U_{2}(\lambda)^{-1}=\phi(\boldsymbol{q}, M(\lambda) x) .
\end{aligned}
$$

$U_{2}(\lambda)$ does commute with the internal symmetries $G(\lambda)$.

If we ask what spins are contained in this representation of the Lorentz group we find

$$
U_{2}(R) a^{\dagger}(\boldsymbol{q}, \mathbf{0}) U_{2}(R)^{-1}=a^{\dagger}(\boldsymbol{q}, \mathbf{0})
$$

and thus, for each $\boldsymbol{q}, a^{\dagger}(\boldsymbol{q}, \mathbf{0})$ transforms like a spin zero particle. While in the first representation $U(\lambda)$ we have particles with all integral spins, in the representation $U_{2}$ we have only scalar particles.

In fact, in the example of an infinite component field given by Streater [14] which violates the correct connection between spin and statistics, the action of the Lorentz group can also be redefined in such a way that there are only scalar particles in the theory and they have the correct connection between spin and statistics.

We have thus shown that if one allows infinitely degenerate particle multiplets one cannot expect the internal symmetry group to have the simple properties shown in the preceding sections, and in such cases, the representation of the Lorentz group itself may not be uniquely defined.

Acknowledgements. I acknowledge many valuable discussions concerning local symmetries with Prof. E. H. Wichmann and Mr. I. Herbst at Berkeley.

I whish to thank Prof. R. Haag for his hospitality at the Institut and for discussions. I also wish to thank the other members of the Institut, particularly Dr. M. Rinke and Mr. F. Perez, for helpful conversations.

I thank my wife, Shyh-rong, for her help and encouragement.

\section{References}

1. Dyson, F. J.: Symmetry groups in nuclear and particle physics. New York: W. A. Benjamin, Inc. 1966.

2. For other discussions of local symmetries, see Doplicher, S., Haag, R., Roberts, J. E.: Commun. Math. Phys. 13, 1 (1969); Robinson, D. W.: In: Symmetry principles and fundamental particles. Ed. by Kursunoglu and Perlmutter. San Francisco: W. H. Freeman and Co. 1967; Lopuszanski, J. T.: Commun. Math. Phys. 14, 158 (1969).

3. Landau, L. J., Wichmann, E. H.: J. Math. Phys. 11, 306 (1970). 
4. Zeeman, E. C.: J. Math. Phys. 5, 490 (1964).

5. Araki, H., Haag, R.: Commun. Math. Phys. 4, 77 (1967).

6. Haag, R.: Phys. Rev. 112, 669 (1958).

7. For other discussions of the linear action of a local symmetry we refer to Doplicher, et al. reference [2] and J. T. Lopuszanski, DUBNA JINR-E-2-4832 (1969).

8. See the discussion of Wightman, A. S.: In: Dispersion relations and elementary particles. Ed. by DeWitt and Omnes. New York: John Wiley Inc. 1960; and Vladimirov, V. S.: Methods of the theory of functions of many complex variables Cambridge: M. I. T. Press 1966.

9. References [8] and lemma 1 of Landau, L. J.: Commun. Math. Phys. 13, 246 (1969).

10. Schwartz, J.: J. Math. Phys. 2, 271 (1961).

11. Weinberg, S.: Phys. Rev. 133, B 1318 (1964).

12. Carruthers, P.: Phys. Rev. Letters 18, 353 (1967).

13. Orzalesi, C. A., Sucher, J., Woo, C. H.: Phys. Rev. Letters 21, 1550 (1968).

14. Streater, R. F.: Commun. Math. Phys. 5, 88 (1967).

Lawrence J. Landau

II. Institut für Theoretische Physik

der Universität Hamburg

D-2000 Hamburg 50

Luruper Chaussee 149 